\title{
Mathematical Modeling for Optimal Design of In-Building Distributed Antenna Systems
}

\author{
Lei Chen and Di Yuan
}

\section{Linköping University Post Print}

\section{Tweet}

N.B.: When citing this work, cite the original article.

Original Publication:

Lei Chen and Di Yuan, Mathematical Modeling for Optimal Design of In-Building Distributed Antenna Systems, 2013, Computer Networks, (57), 17, 3428-3445.

http://dx.doi.org/10.1016/j.comnet.2013.07.027

Copyright: Elsevier

http://www.elsevier.com/

Postprint available at: Linköping University Electronic Press

http://urn.kb.se/resolve?urn=urn:nbn:se:liu:diva-89716 


\title{
Mathematical modeling for optimal design of in-building distributed antenna systems
}

\author{
Lei Chen*, Di Yuan \\ Department of Science and Technology, Linköping University, Sweden
}

\begin{abstract}
In-building Distributed Antenna System (IB-DAS) has proven to be one of the most promising In-Building Solutions (IBS) to provide coverage and capacity for indoor users. We consider optimal deployment of the passive IB-DAS, focusing on mathematical optimization models based on integer programming, for the topology design and optimal equipment selection of IB-DAS. The models minimize the cable cost and keep the transmit power at each antenna within a given interval defined for coverage and interference control. The models can deliver optimal solutions to systems of which the size is of practical relevance. To improve the time efficiency, we develop preprocessing techniques that integrate the building layout data into the system modeling. Application of the models to realistic IB-DAS deployment demonstrates the effectiveness of the models.
\end{abstract}

Keywords: distributed antenna systems, integer programming, optimization

\section{Introduction}

Providing sufficient coverage and capacity for indoor users has always been a challenge for the mobile operators. With the evolution of cellular technologies, in-building traffic has grown dramatically. Today, over $80 \%$ of mobile data traffic and $70 \%$ of voice traffic are generated by in-building users [1]. The fast development and deployment of the next generation cellular networks, such as Long Term Evolution (LTE) will push the in-building traffic to an even higher level, making it the main revenue generator for the mobile

\footnotetext{
${ }^{*}$ Corresponding author
} 
operators. Thus, it has become crucial for the operators to deploy dedicated in-building systems to fulfill the increasing indoor data requirements.

The demand of in-building users is a performance bottleneck for the overall performance of cellular networks, and insufficient in-building coverage contributes largely to the degradation of the overall network throughput of the macro-cells $[2,3]$. For in-building users, the main reason for the poor signal level is due to the loss of signal strength for wall penetration. The loss value is within the range of 20 and $50 \mathrm{~dB}$ [9]. With the migration from lower frequency bands of GSM (e.g., $800 \mathrm{MHz}$ and $900 \mathrm{MHz}$ ) to higher frequency bands of LTE (e.g., $2.4 \mathrm{GHz}$ ), the signal loss from wall penetration becomes higher. For a concrete wall that results in $20 \mathrm{~dB}$ penetration loss for GSM at $800 / 900 \mathrm{MHz}$, the corresponding loss for LTE is more than $30 \mathrm{~dB}$ and hence significantly higher. The existence of metals in the wall, such as lead, introduces further attenuation. In addition to wall penetration, the orthogonality of the radio channels can be compromised because of multi-path reflections for the in-building coverage of Universal Mobile Telecommunications System (UMTS) networks. In-building Distributed Antenna System (IB-DAS) has proven to be one of the most promising methods for dedicated in-building solutions. With a properly deployed IB-DAS system, a favorable link budget, i.e., better radio signal reception, can be provided because of the absence of wall penetration requirement and less impact of multi-path reflections. Also, coverage holes can be easily avoided by a careful planning of the distributed antennas, as well as the use of directional antennas. As a result, in-building users will experience a satisfactory level of network presence. Besides, IBDAS can help offload the macro-cell network traffic significantly. In addition, the average downlink transmission power of the base station can be reduced, making more system capacity available to macro-cells for serving outdoor users. Uplink transmission power can also be reduced significantly so that a much longer battery life can be achieved for the user equipments.

A major type of IB-DAS is Passive DAS (P-DAS). In this paper, we consider optimal deployment of P-DAS. A typical P-DAS consists of a center base station (BS) and a number of distributed antennas. The BS uses a donor antenna on the roof of the building to connect to the macro-cell for regular communication service as well as to specialized public safety site for emergency service. Distributed antennas are connected to the center BS via copper coaxial cable. Power equipments, such as splitters and taps, are used to split and distribute the power towards the antennas. P-DAS has been extensively used for cost-effective indoor access in small buildings. 
Previous works of DAS planning mostly concentrate on the performance gains brought by the deployment of DAS for the in-building scenarios. In [4], the authors study the performance gain of DAS both by analytical reasoning and system simulations. The work in [5] compares the results of the DAS system with a conventional Array Antenna System (AAS). The author of [6] discusses the importance of a dedicated in-building solution for 3G networks. In [7], the authors propose to use repeaters based on DAS deployment for capacity improvement of UMTS networks. All these works have pointed out the importance of a proper deployment of IB-DAS in order to bring significant performance gains to in-building users. An optimization-oriented study of PDAS deployment is provided in [8]. The DAS Forum [9] has dedicated itself to the promotion and development of the DAS solutions, and provides case studies of DAS in various fields, e.g., regular data services, health care, public safety, and so on.

From the planning point of view, deployment of IB-DAS systems amounts to constructing an optimal topology which connects all distributed antennas to the center BS, and selecting equipments at the intermediate nodes. To guarantee the performance, the output power of each antenna, which is determined by both the topology and equipment selection, is confined to be a pre-defined interval. Cable usage, which contributes to the most of the overall cost of the deployment of IB-DAS, is minimized. We approach the problem from a mathematical programming standpoint, and develop mixed integer programming models for problem solution. Preprocessing techniques utilizing the building structure have been introduced to strengthen the computational efficiency of the models. We present numerical results demonstrating that our solution approach enables the optimal deployment solution of IB-DAS systems of realistic size.

The rest of the paper is organized as follows. Section 2 gives a detailed presentation of the P-DAS. Section 3 introduces notation, and performs system modeling of DAS deployment. Two mixed integer linear programming models are presented and detailed in Sections 4 and 5, respectively. Preprocessing techniques for enhancing the computational efficiency are developed in Section 6. Section 7 presents performance evaluation of the solution approach for realistic IB-DAS deployment scenarios. Section 8 concludes the paper and discusses future works. 


\section{Distributed antenna system}

Figure 1 illustrates the main components of a typical P-DAS. As can be seen from the figure, a P-DAS consists of the following components:

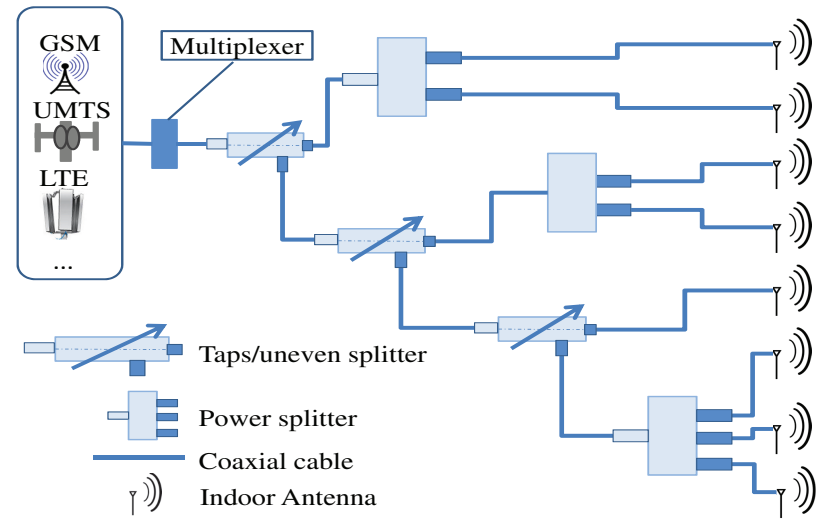

Figure 1: Conceptual illustration of P-DAS.

- Center BS: BS is the power source for all antennas. BS can be based on any of the access technologies such as GSM, UMTS, HSPA as well as LTE. When the BS has multiple donor antennas for different access technologies, the signals will be multiplexed before being fed into the IB-DAS system.

- Coaxial cable: Coaxial cable has been widely used for IB-DAS systems. There are several standard cables that can be used for IB-DAS deployment. Once the radio frequency and cable type are chosen, the signal loss over the cable will purely depend on its length.

- Power splitters: Splitters are the commonly used components in IBDAS to divide the incoming signal into multiple signal streams. The term usually refers to the equipment type that distributes the power evenly over the output ports. For example, in the case of two output ports, each will have half of the input power. Besides the power split, there usually exists a certain insertion loss at each of the output ports, which should be considered during the implementation. 
- Power taps: If all antennas have similar range of coverage, it is desirable that they have roughly the same output power [10]. The usage of power splitters may not be enough to achieve this because in many cases some distributed antennas are very near to the BS whereas others are far away. Taps, also referred to as uneven splitters, are used to tap a small portion of the power along the cable and leave the rest for serving antennas located further away. The installation of power splitters and taps is a key issue in IB-DAS planning as it plays the role of distributing the power for the purpose of coverage and capacity guarantee.

- Distributed antennas: Distributed antennas are the terminals of the IB-DAS. They serve as the access points for the in-building users. The location and desired output power of the antennas are typically determined from a radio access planning tool that, based on expected user distribution and traffic demand, selects the locations and the power levels such that sufficient coverage is provided and the interference between the antennas is acceptable. The output power levels are thus part of the input to topology design and equipment selection. In most of the cases, it is not necessary or even possible to meet the target level exactly. Usually, a threshold can be used to limit the power deviation of each antenna.

\section{System modeling}

IB-DAS design is an optimization problem over a graph. There are two sets of nodes in addition to the BS node. The first set consists of the distributed antennas. The second set is composed by candidate locations, referred to as intermediate nodes henceforth, for power equipment installation. The locations of the intermediate nodes are pre-chosen by considering the building structure as well as the easiness for equipment installation. The topology of an IB-DAS is a rooted tree originating from the BS and connects all distributed antennas through cables and power equipments, see Figure 2 for an illustration.

To represent the graph, we introduce the following notation. We denote the BS by $s$ and the set of intermediate nodes by $\mathcal{N}$. The set of antennas is denoted by $\mathcal{T}$. For each antenna $t \in \mathcal{T}, p_{t}^{t a r}$ denotes the target output power. All distributed antennas will connect to the BS through the intermediate 


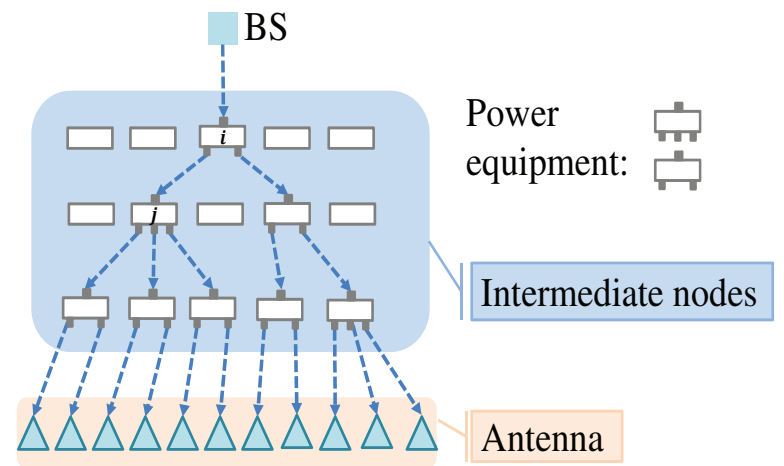

Figure 2: A graph illustration of IB-DAS.

nodes, where power equipments can be installed. The connections form a tree with the BS as the root. The leaves are $\mathcal{T} \cup\{s\}$, because the connections from the BS terminate at the nodes in $\mathcal{T}$, and the BS itself has no power equipment installed. All the intermediate nodes that are chosen to be part of the tree will have power equipments. Note that there is no direct link between the BS and any of the antennas. Thus the path from the BS to an antenna consists in three segments of arcs: an arc from the BS to an intermediate node, one or multiple arcs between the intermediate nodes, and one arc connecting the last intermediate node to the antenna. We denote by $\mathcal{A}$ the set of potential arcs to be used. The length of arc $(i, j)$ is denoted by $d_{i j}$. The set of edges is denoted by $\mathcal{E}$. An edge $(i, j) \in \mathcal{E}$ implies two arcs $(i, j)$ and $(j, i)$ in $\mathcal{A}$. Not all arcs are necessarily of relevance to topology design. For example, if the tree orientation is considered going from the root to the antennas, then arcs connecting the antennas to the intermediate nodes can be discarded. For notational convenience, however, we do not introduce restriction to the arc set at this stage. How this aspect affects mathematical modeling will be detailed later.

For calculating antenna output power, the per-meter cable loss in $\mathrm{dB}$ is denoted by $\xi$, which is a positive number throughout the paper. We denote the set of power equipment types by $\mathcal{H}$ including both splitters and taps. An equipment type is characterized by the number of output ports and the loss values of the ports (e.g., a positive value of $3 \mathrm{~dB}$ loss means the power is decreased by half). We denote the discrete set of all potential port losses by $\mathcal{R}$ (in $\mathrm{dB}$ ); this is the union set of the loss values over all equipment types. An element $r \in \mathcal{R}$ is referred to as a port type (which may appear on 
multiple equipment types) with $r \mathrm{~dB}$ loss. To denote the relation between port loss $r \in \mathcal{R}$ and each of the power equipment types $h \in \mathcal{H}$, we introduce a parameter $\alpha_{r h}, r \in \mathcal{R}, h \in \mathcal{H}$ which specifies how many ports (possibly zero) with loss $r \in \mathcal{R}$ are present for equipment type $h$.

In the deployment of IB-DAS, coaxial cable cost usually dominates the total expenditure. Thus the optimal deployment should use a minimum amount of cable. In addition, from the DAS performance viewpoint, the deployment is driven by the power targets of the antennas. Having output powers close to the targets ensures the coverage and capacity for the in-building users for each antenna, and keeps the signal leakage to outdoor users at a minimum level. In many cases, it is not possible to meet exactly the power targets using the cable connections and power equipment types at choice. One way of system modeling is to minimize the deviation from the power targets, and weight this quantity in the objective function. However, finding a proper weight is far from trivial. Furthermore, a potential problem is the result of a very uneven power distribution among the in-building antennas, leading to significant coverage holes and signal leakage. In this paper, we apply a threshold, denoted by $p_{t}^{t h r}(t \in \mathcal{T})$, to bound the absolute difference between the power target and the achieved value. If the threshold is too stringent, the topology design and equipment type selection problem may be infeasible. To deal with such a case, the problem can be solved with several threshold values to generate multiple candidate design solutions.

Optimal deployment of IB-DAS amounts to designing a tree having the BS $s$ as the root and the antennas as the leaves, and the selection of an equipment type for each intermediate tree node as well as a port assignment for the outgoing arcs of the node. The objective of the deployment is to minimize the total cable cost, with constraints representing the power deviation threshold. There is no doubt that the problem is NP-hard. Indeed, the well-known and NP-hard Steiner arborescence problem [11] reduces to the IB-DAS deployment problem. A sketch of the reduction consists in the following. First, for each terminal node of the Steiner arborescence problem, there is one antenna to which the terminal node provides the only possible direct connection. Second, an extra node is introduced for the source (which is restricted to have degree one in IB-DAS). The extra node, to be connected with intermediate nodes, is the only possible connection from the source. Third, the power threshold is large enough to be redundant and there is no restriction on the number of ports, i.e., there is one equipment type available for each possible out-degree. After the reduction, the two problems become 
equivalent, and the NP-hardness of IB-DAS deployment follows. For our practical application with IB-DAS planning scenarios where the size is naturally set by the deployment process (e.g., the design of one floor is typically de-coupled from that of other floors), striving for optimality with a reasonable amount of computational effort is not only desirable, but also potentially feasible by means of integer programming.

From the discussion thus far, it is clear that optimal IB-DAS deployment is a type of network design problem, for which mathematical modeling and integer linear programming have been successfully used in various applications $[12,13,14,15,16,17]$. On the other hand, the IB-DAS optimization problem has its peculiarities, in particular the tasks of equipment selection and port assignment, that are not present in classical network design applications. In this paper, our goal is to apply advanced integer programming modeling to enable to approach optimal or near-optimal solutions for IB-DAS deployment for planning scenarios of practical size. We develop two modeling approaches relying on very different concepts. The first uses network flows to enforce connection from the source to the antennas, and the second is based on the idea that undirected Steiner tree can be viewed as a number of Steiner arborescences.

\section{Flow formulation (DAS-F)}

The notion of source-destination flow is commonly used for formulating network design problems. In this section, we develop a flow formulation, denoted by DAS-F, for IB-DAS deployment. In DAS-F, the arc set $\mathcal{A}$ contains arcs from the BS to the intermediate nodes, arcs between the intermediate nodes, and finally the arcs from the intermediate nodes to the antennas, see Figure 3 for an illustration. Arcs from the antennas to the intermediate nodes as well as arcs from the intermediate nodes to the BS are discarded in the model, because the flow goes only in the direction from the BS to the antennas.

\subsection{Decision variables}

In DAS-F, the tree topology is enforced by introducing a unit of flow from the BS to each antenna. Additional design decisions include the selection of equipment (splitters or taps) with a specific type for each intermediate tree node, and the mapping between the ports and the outbound arcs. To these effects, we introduce the following four sets of variables. 


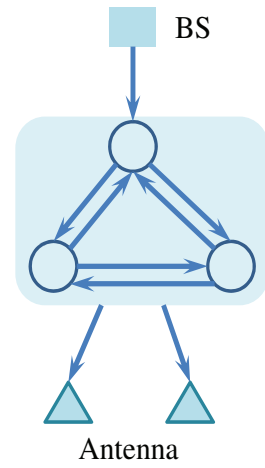

Figure 3: Graph illustration for the set of arcs for DAS-F.

$$
\begin{aligned}
& x_{i j}^{t}= \begin{cases}1 & \text { if arc }(i, j) \in \mathcal{A} \text { carries the flow } \\
\text { from the BS to antenna } t \in \mathcal{T}, \\
0 & \text { otherwise. }\end{cases} \\
& y_{i j}^{r}= \begin{cases}1 & \text { if arc }(i, j) \in \mathcal{A} \text { is assigned a port type } r \in \mathcal{R}, \\
0 & \text { otherwise. }\end{cases} \\
& z_{i h}= \begin{cases}1 & \text { if intermediate node } i \in \mathcal{N} \\
0 & \text { has equipment } h \in \mathcal{H} \text { installed, }\end{cases} \\
& p_{i}=\text { power of node } i, i \in\{s\} \cup \mathcal{N} \cup \mathcal{T} .
\end{aligned}
$$

\subsection{Constraints}

To ease the presentation, the constraints are grouped into three categories. These are defined for topology design, equipment selection and the power calculation, respectively.

- Topology design: The connectivity between the BS node and the antennas is achieved by means of multi-commodity flows for communication networks [18]. Here, multi-commodity flow refers to multiple flows, sharing arcs but differ in their destinations (i.e., antennas). The flow for each antenna defines partially the topology. In addition, the decisions of topology design and power loss values are inherently coupled with each other. In the following, we define constraints for topology design and its coupling with variables representing power loss. 
Constraints (F.5) require that for any antenna $t$, there is exactly one unit of flow sent from the source BS node. As there is no power equipment at the BS, all flows originating from the BS will share a common arc. This is modeled by constraints (F.6). Each antenna is the destination of exactly one unit of flow, as specified by (F.7). Constrains (F.8) define flow conservation at the intermediate nodes, that is, the incoming flow and outgoing flow of each antenna must be in balance. Constraints (F.9)-(F.11) represent the coupling relation between topology and the presence of power loss. By (F.9), at least one port type will be chosen for arc $(i, j)$, if the flow towards antenna $t$ goes through the arc. Conversely, if a port type is selected for arc $(i, j)$, then there must be at least one antenna $t$ whose flow passes this arc, as stated by constraints (F.10). Constraints (F.11) are the equality form of (F.9) for arcs having antenna as their end nodes. The equality sign stems from the facts that, for any antenna $t$, exactly one of the incoming arcs must be chosen, and one power loss value must be selected for the starting node of the arc. Finally, constraints (F.12) state that for each node $j$, equipment power loss occurs on at most one incoming arc. As a result, the flows will be constrained on a tree topology.

$$
\begin{array}{ll}
\sum_{j \in \mathcal{N}:(s, j) \in \mathcal{A}} x_{s j}^{t}=1, & \forall t \in \mathcal{T} \\
x_{s j}^{t_{1}}=x_{s j}^{t_{2}}, & \forall t_{1}, t_{2} \in \mathcal{T}, j \in \mathcal{N}: \\
\sum_{j \in \mathcal{N}:(j, t) \in \mathcal{A}} x_{j t}^{t}=1, & t_{1} \neq t_{2},(s, j) \in \mathcal{A} \\
\sum_{j \in \mathcal{N}:(j, i) \in \mathcal{A}} x_{j i}^{t}=\sum_{j \in \mathcal{N}:(i, j) \in \mathcal{A}} x_{i j}^{t}, & \forall t \in \mathcal{T} \\
x_{i j}^{t} \leq \sum_{r \in \mathcal{R}} y_{i j}^{r}, & \forall(i, j) \in \mathcal{A}, t \in \mathcal{T}, i \neq s \\
y_{i j}^{r} \leq \sum_{t \in \mathcal{T}} x_{i j}^{t}, & \forall(i, j) \in \mathcal{A}, r \in \mathcal{R}, i \neq s \\
x_{i t}^{t}=\sum_{r \in \mathcal{R}} y_{i t}^{r}, & \forall i \in \mathcal{N}, t \in \mathcal{T}:(i, t) \in \mathcal{A}
\end{array}
$$




$$
\sum_{i \in \mathcal{N}:(i, j) \in \mathcal{A}} \sum_{r \in \mathcal{R}} y_{i j}^{r} \leq 1, \quad \forall j \in \mathcal{N}
$$

- Power equipment selection: Power equipment selection involves both equipment type and the port-to-arc assignment for each intermediate node of the tree. The following constraints ensure that, if any flow passes through an intermediate node, then an equipment type has to be selected and all the ports are used.

$$
\begin{array}{ll}
\sum_{h \in \mathcal{H}} z_{i h} \leq 1, & \forall i \in \mathcal{N} \\
\sum_{r \in \mathcal{R}} y_{i j}^{r}+\sum_{r \in \mathcal{R}} y_{j i}^{r} \leq 1, & \forall i, j \in \mathcal{N},(i, j) \in \mathcal{A} \\
\sum_{j \in \mathcal{N} \cup \mathcal{T}:(i, j) \in \mathcal{A}} y_{i j}^{r}=\sum_{h \in \mathcal{H}} z_{i h} \alpha_{r h}, & \forall i \in \mathcal{N}, r \in \mathcal{R}
\end{array}
$$

By (F.13), at most one equipment type can be present at any intermediate node. For any two intermediate nodes $i, j \in \mathcal{N}$, only one of the two $\operatorname{arcs}(i, j)$ and $(j, i)$ will be used to route the flow from the source, and thus at most one of the two ends will have equipment power loss. This is defined by constraints (F.14). Next, constraints (F.15) give the effect that all ports of an installed equipment are used for connections. Note that at most one $z$-variable in the right-hand side of (F.15) can be one. Thus, for each node $i$ and possible power loss value $r$, the righthand side of (F.15) is the total number of outgoing ports (possibly zero) with loss $r$ at node $i$. This number must be equal to the number of outgoing arcs that are assigned the corresponding value. The latter is given by the sum in the left-hand side.

- Power constraints: The last category of constraints is used to apply power calculation in connection with the other decisions made, along with enforcing the power deviation threshold of the antennas. Recall that the power loss occurs along the cables and at the equipment ports. For any of the two nodes $i, j \in \mathcal{N} \cup \mathcal{T}$ and the arcs between them, the power loss can be calculated as follows. 


$$
p_{j}=p_{i}-\xi d_{i j}-r, \text { if and only if } y_{i j}^{r}=1
$$

By (F.16), the power of node $j$ equals that of node $i$ minus the distancebased cable loss and the loss caused by $i$ 's equipment with connection to $j$, if the corresponding $y$-variable is one. Because only one equipment loss value is assigned to any arc by (F.12), the equation can be rewritten over all possible equipment loss values as follows.

$$
p_{j}=p_{i}-\xi d_{i j} \sum_{r \in \mathcal{R}} y_{i j}^{r}-\sum_{r \in \mathcal{R}} r y_{i j}^{r}, \quad \text { if and only if } \sum_{r \in \mathcal{R}} y_{i j}^{r}=1
$$

Power calculation for arcs leaving the BS has to be performed in a slightly different way, for the reason that no equipment is installed at the $\mathrm{BS}$, for which no $y$-variable is present. Instead, the $x$-variables are used to set the condition for power calculation, and the loss originates from the arc length only, as formulated below. Note that the power of the source node $p_{s}$ is also a variable.

$$
p_{j}=p_{s}-\xi d_{s j}, \quad \text { if and only if } x_{s j}^{t}=1, \forall t \in \mathcal{T}
$$

In the IB-DAS tree topology, exactly one arc from the BS is selected. The selected arc carries flows towards all $|\mathcal{T}|$ antennas. Thus, if any $\operatorname{arc}(s, j), j \in \mathcal{N}$ is chosen, all flows go through that arc, meaning that $\sum_{t \in \mathcal{T}} x_{s j}^{t}=|\mathcal{T}|$. Thus, the power can be calculated as follows for the arcs from the BS.

$$
p_{j}=p_{s}-\frac{\sum_{t \in \mathcal{T}} \xi d_{s j} x_{s j}^{t}}{|\mathcal{T}|}, \text { if and only if } \sum_{t \in \mathcal{T}} x_{s j}^{t}=|\mathcal{T}|
$$

Equations (F.17) and (F.19) are non-linear, because of the if-and-onlyif statement. To reach linearity, the equality is represented by two inequalities, and a parameter $M$, referred to as big $\mathrm{M}$, is used. The linear reformulations for power calculation are presented below. 


$$
\begin{aligned}
& p_{j} \leq p_{i}-\xi d_{i j} \sum_{r \in \mathcal{R}} y_{i j}^{r}-\sum_{r \in \mathcal{R}} r y_{i j}^{r}+M\left(1-\sum_{r \in \mathcal{R}} y_{i j}^{r}\right), \\
& \forall i, j \in \mathcal{N} \cup \mathcal{T}:(i, j) \in \mathcal{A} \\
& p_{j} \geq p_{i}-\xi d_{i j} \sum_{r \in \mathcal{R}} y_{i j}^{r}-\sum_{r \in \mathcal{R}} r y_{i j}^{r}-M\left(1-\sum_{r \in \mathcal{R}} y_{i j}^{r}\right) \text {, } \\
& \forall i, j \in \mathcal{N} \cup \mathcal{T}:(i, j) \in \mathcal{A} \\
& p_{j} \leq p_{s}-\frac{\xi d_{s j} \sum_{t \in \mathcal{T}} x_{s j}^{t}}{|\mathcal{T}|}+M\left(1-\frac{\sum_{t \in \mathcal{T}} x_{s j}^{t}}{|\mathcal{T}|}\right), \\
& \forall j \in \mathcal{N}:(s, j) \in \mathcal{A} \\
& p_{j} \geq p_{i}-\frac{\xi d_{s j} \sum_{t \in \mathcal{T}} x_{s j}^{t}}{|\mathcal{T}|}-M\left(1-\frac{\sum_{t \in \mathcal{T}} x_{s j}^{t}}{|\mathcal{T}|}\right), \\
& \forall j \in \mathcal{N}:(s, j) \in \mathcal{A}
\end{aligned}
$$

In (F.20), the power of node $j$ is bounded from above by the power calculation in (F.18), if $\sum_{r \in \mathcal{R}} r y_{i j}^{r}=1$. Otherwise the last term will be at least $M$, and the inequality does not pose any constraint on the power of $j$ if $M$ is sufficiently large (e.g., the sum of the cable loss of all potential arcs). Constraints (F.21) are similar in construction, with the effect of bounding the power of $j$ from below if $\sum_{r \in \mathcal{R}} r y_{i j}^{r}=1$. Thus these two constraints together achieve the effect of (F.18). The following two constraints carry the same notion for replacing (F.19). The power constraints become complete by setting the threshold requirement at the antennas, as stated below.

$$
\begin{array}{ll}
p_{t}^{t h r} \geq p_{t}^{t a r}-p_{t}, & \forall t \in \mathcal{T} \\
p_{t}^{t h r} \geq p_{t}-p_{t}^{t a r}, & \forall t \in \mathcal{T}
\end{array}
$$

\subsection{Objective function}

The objective function is to minimize the total amount of cabling. This is formulated below. 


$$
\sum_{(s, j) \in \mathcal{A}} \sum_{t \in \mathcal{T}} \frac{d_{s j} x_{s j}^{t}}{|\mathcal{T}|}+\sum_{(i, j) \in \mathcal{A}: i \neq s} d_{i j} \sum_{r \in \mathcal{R}} y_{i j}^{r}
$$

In (F.26), the first term is the cable length from the BS. Because all flows will be on a single arc from the BS, the sum is divided by $|\mathcal{T}|$ to obtain the cable length. The second term gives the total cable length originating from the intermediate nodes.

\subsection{Model summary}

Putting the pieces together, optimal deployment of IB-DAS using network flows can be made by the following mixed integer linear programming formulation.

$$
\begin{array}{rrr}
{[\mathbf{D A S}-\mathbf{F}]:} & \min (\mathrm{F} .26) \\
& \text { s.t. } \quad(\text { F.6 })-(\text { F.15), (F.20)-(F.23), (F.24), (F.25). }
\end{array}
$$

With the notion of network flow, formulation DAS-F is fairly straightforward. However, its applicability for problem solving is limited to small-scale planning scenarios. The main reason is the presence of the big-M parameter, making the continuous relaxation of DAS-F rather weak in approximating the integer optimum.

\section{Multi-tree formulation (DAS-MT)}

Our second modeling approach, which explores the concept of multi-tree, is less trivial than the use of network flows. Recall that the topology of IB-DAS assembles a Steiner tree, with the difference that all antennas in ID-DAS must be tree leaves. An undirected Steiner tree can be viewed as many Steiner arborescences with different root nodes [19, 20]. Moreover, the multiple arborescences can be coupled together to share a common tree topology. Applying the concept, for IB-DAS the topology can be viewed as $1+|\mathcal{T}|$ arborescences rooted at the BS and the antennas, respectively. These are the leaves in the common tree. Hereafter, we refer to the model based on the concept of multi-tree as DAS-MT.

DAS-MT uses both the edge set $\mathcal{E}$ and the arc set $\mathcal{A}$. The definition of the set of arcs is slightly different from that for DAS-F. Specifically, arcs adjacent 
to the BS are reversed. Thus only arcs having BS and the antennas as the end nodes are included in set $\mathcal{A}$ in DAS-MT. The arcs between intermediate nodes remain. The composition is illustrated in Figure 4.

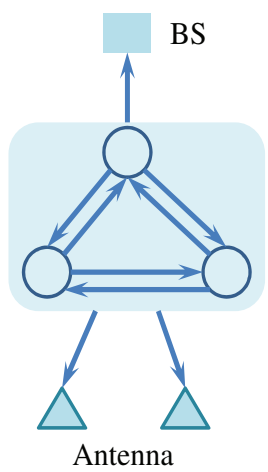

Figure 4: Graph illustration of the arcs in DAS-MT.

\subsection{Decision variables}

In DAS-F, the flow on each arc and the port type choice of each arc are denoted by $x_{i j}^{t}$ and $y_{i j}^{r}$, respectively. In DAS-MT, the key variables, as defined below, consider the joint effect of arc use and port type selection.

$$
q_{i j}^{r k}= \begin{cases}1 & \text { if arc }(i, j) \text { is used to reach node } k \text { with port type } r \\ & \text { for node } k \in\{s\} \cup \mathcal{T} \backslash\{j\} \\ 0 & \text { otherwise }\end{cases}
$$

The rationale of variable $q_{i j}^{r k}$ is to specify the orientation of using arc $(i, j)$ to reach node $k$ in the tree, along with the port type for $\operatorname{arc}(i, j)$. The variables are defined for reachability of $s$ and $\mathcal{T}$ (the BS and the antenna) that must be part of the topology design. Note that superscript $k$ equals subscript $j$ for some of the $q$-variables, i.e., variables $q_{i j}^{r j},(i, j) \in \mathcal{A}, r \in \mathcal{R}$. If $(i, j)$ is part of the tree, then clearly $q_{i j}^{r j}=1$ for some $r \in \mathcal{R}$. Thus the variable will in effect specify equipment selection, as will be detailed later.

For any edge connecting two intermediate nodes, both directions will be used (i.e., $q$-variables have value one) if the edge is part of the IB-DAS tree. By the definition of the $q$-variables, it is required that the port type is provided at the starting node of both arcs. In reality, however, an intermediate 


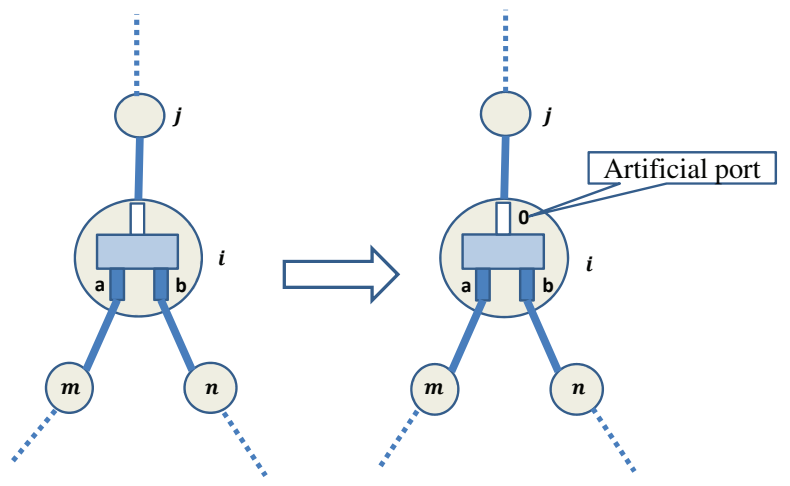

Figure 5: Introduction of the artificial port.

node does not have a port loss for an incoming arc. To deal with this issue, our modeling solution is to introduce an artificial outgoing port type associated with the input for all equipments types. The notion is illustrated in Figure 5. In the left part of the figure, the real equipment installed at node $i$ has two output ports, with power loss $a$ and $b$, respectively. Hence for nodes $m$ and $n$, and all other nodes $k, k \in \mathcal{N} \cup \mathcal{T}$ connected to $i$ through $m$ and $n, q_{i m}^{a k}=1$ or $q_{i n}^{b k}=1$. For a node $k$ that is reached in the direction from $i$ to $j$, the output port is in fact the incoming port of the equipment of $i$. We generalize the equipment definition by adding an artificial outgoing port type with zero dB loss, referred to as type-0 port, at the input side of equipment type, as shown in the right part of the figure, with the effect that $q_{i j}^{0 k}=1$ for any node $k$ reached via $(i, j)$. Note that for this example, node $i$ reaches the BS node $s$ via $j$. Thus $q_{i j}^{0 s}=1$.

Besides the consideration of a type-0 outgoing port for every equipment type, we introduce an artificial equipment type. The equipment type has a single type- 0 output port, and, similar to the other equipment types, a type-0 output port on the incoming side. The reason of introducing the artificial equipment type is similar to that for including the type- 0 port. Namely, some intermediate nodes in the tree may have a degree of two, and for such nodes an output loss value is necessitated by the definition of the $q$-variables. Installing an artificial equipment, which corresponds to no real equipment selection, meets this requirement. The aspect is illustrated for node $i$ in the example shown in Figure 6 . Note that the use of artificial equipment can be avoided here, if edge $(j, n)$ is present in the underlying graph. However, with such "shortcut" edges, the graph size becomes prohibitively high for optimization. 


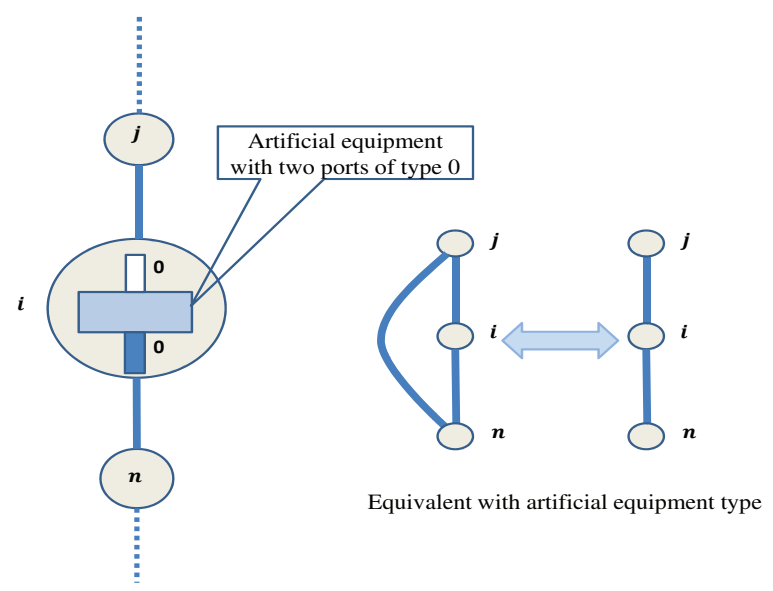

Figure 6: Introduction of artificial equipment.

Thus the artificial equipment type eliminates the need of including additional edges without compromising the solution space.

DAS-MT uses other design variables in addition to the multi-tree $q$ variables to specify a tree topology. The additional variables are defined below. Note that we use $\bar{y}$ instead of $y$ to differentiate DAS-MT from DASF for the sake of clarity, even though these variables sets have the same meaning.

$$
\begin{aligned}
\bar{y}_{i j} & = \begin{cases}1 & \text { if edge }(i, j) \in \mathcal{E} \text { exists in the solution tree } \\
0 & \text { otherwise }\end{cases} \\
w_{i} & = \begin{cases}1 & \text { if node } i \in \mathcal{N} \text { exists in the solution tree } \\
0 & \text { otherwise }\end{cases}
\end{aligned}
$$

For equipment selection, we define variables $\bar{z}$ for DAS-MT. They act as the $z$-variables in DAS-F, with the slight change in notation for differentiation.

$$
\bar{z}_{i h}= \begin{cases}1 & \text { if intermediate node } i \in \mathcal{N} \text { has } \\ & \text { equipment type } h \in \mathcal{H} \text { installed } \\ 0 & \text { otherwise }\end{cases}
$$


The variables defined thus far do not indicate if $(i, j)$ is in the path from the BS to an antenna $t$ or not. This indication is necessary for power calculation. To identify the paths from the BS, the following variables are used.

$$
\bar{x}_{i j}^{t}= \begin{cases}1 \quad \begin{array}{l}
\text { if edge }(i, j) \in \mathcal{E} \text { exists in the path } \\
\text { from the BS to antenna } t \in \mathcal{T}
\end{array} \\
0 \quad \text { otherwise }\end{cases}
$$

Unlike $x_{i j}^{t}$ in DAS-F, $\bar{x}_{i j}^{t}$ is defined for edges instead of arcs. As will be clear later on, the power of each antenna can be expressed without explicitly specifying which direction of an edge is used for the path from the BS. The last set of variables are continuous power variables. For DAS-MT, power variables are required only for the BS and the antennas, as defined below.

$$
p_{i}=\text { power of node } i, i \in\{s\} \cup \mathcal{T} \text {. }
$$

\subsection{Constraints}

Following the description style for DAS-F, the constraints for DAS-MT are grouped into four categories. The first three categories are defined for topology design, equipment selection, and power calculation, respectively. The fourth category of constraints, referred to as path identification constraints, is used to identify the edges forming the path from the BS to each antenna, in order to facilitate power calculation.

- Topology design: The constraints for topology design in DAS-MT are provided below. Together they ensure the edges chosen indeed form a tree that connects all the leaf nodes (the BS and antennas) via intermediate nodes. We remark that the number of constraint sets is not as high as it may appear at a first glance, because many of them are special cases of others.

Constraints (MT.7) state a necessary condition of a tree topology, namely the number of edges is equal to the number of nodes minus one. By the two sets of constraints (MT.8) and (MT.9), the inclusion of any edge $(i, j) \in \mathcal{E}$ in the tree is possible only if both nodes $i$ and $j$ are part of the tree. Note that the BS and antennas must always be in the tree; this is ensured by constraints (MT.10). If an intermediate node is used in the tree topology, then the node must be able to reach 
both the BS with a type-0 port, as well as all the antennas with other port types. These conditions are formulated in constraints (MT.11) and (MT.12).

$$
\begin{array}{ll}
\sum_{(i, j) \in \mathcal{E}} \bar{y}_{i, j}=\sum_{i \in \mathcal{N} \cup\{s\} \cup \mathcal{T}} w_{i}-1 & \\
w_{i} \geq \bar{y}_{i j}, & \forall(i, j) \in \mathcal{E} \\
w_{j} \geq \bar{y}_{i j}, & \forall i, j) \in \mathcal{E} \\
w_{i}=1, & \forall i \in \mathcal{N}\} \cup \mathcal{T} \\
w_{i}=\sum_{j \in \mathcal{N} \cup\{s\}:(i, j) \in \mathcal{A}} q_{i j}^{0 s}, & \forall i \in \mathcal{N}, t \in \mathcal{T} \\
w_{i}=\sum_{j \in \mathcal{N} \cup \mathcal{T}:(i, j) \in \mathcal{A}} \sum_{r \in \mathcal{R}} q_{i j}^{r t}, & \\
w_{s}=\sum_{j \in \mathcal{N}:(j, s) \in \mathcal{A}} q_{j s}^{0 s} & \forall t \in \mathcal{T} \\
w_{t}=\sum_{j \in \mathcal{N}:(j, t) \in \mathcal{A}} \sum_{r \in \mathcal{R}: r \neq 0} q_{j t}^{r t}, & \forall(i, j) \in \mathcal{E}: i, j \in \mathcal{N}, k \in\{s\} \cup \mathcal{T} \\
\sum_{r \in \mathcal{R}} q_{i j}^{r k}+\sum_{r \in \mathcal{R}} q_{j i}^{r k}=\bar{y}_{i j}, & \\
\sum_{r \in \mathcal{R}} q_{i j}^{r j}=\bar{y}_{i j}, & \forall(i, j) \in \mathcal{E}: i, j \in \mathcal{N} \\
\sum_{r \in \mathcal{R}} q_{j i}^{r i}=\bar{y}_{i j}, & \forall(i, j) \in \mathcal{E}: i, j \in \mathcal{N} \\
q_{i j}^{0 s}+q_{j i}^{0 s}=\bar{y}_{i j}, & \forall(i, t) \in \mathcal{E}: t \in \mathcal{T} \\
\sum_{r \in \mathcal{R}} q_{j s}^{r s}=\bar{y}_{s j}, & \forall(i, j) \in \mathcal{E}: i, j \in \mathcal{N} \\
q_{j s}^{0 s}=\bar{y}_{s j}, & \forall(s, j) \in \mathcal{E} \\
\sum_{r \in \mathcal{R}, t \in \mathcal{T}} q_{i s}^{r t}=0, & \forall(s, j) \in \mathcal{E} \\
\sum_{r \in \mathcal{R}: r \neq 0} q_{i t}^{r t}=\bar{y}_{i t}, & \forall(i, s) \in \mathcal{A} .
\end{array}
$$




$$
\begin{array}{ll}
\sum_{\substack{r \in \mathcal{R}, k \in\{s\} \cup \mathcal{T}: k \neq t \\
q_{i j}^{r k} \leq q_{i j}^{r j},}} q_{i t}^{r k}=0, & \forall(i, t) \in \mathcal{A}: t \in \mathcal{T} \\
& \forall(i, j) \in \mathcal{A}, r \in \mathcal{R}, k \in\{s\} \cup \mathcal{T}
\end{array}
$$

The BS and the set of antennas must be connected via intermediate nodes. Constraints (MT.13) model the condition that an intermediate node is connected to the BS with a type-0 port, while constraints (MT.14) model the condition that for each antenna, there is an arc from one of the intermediate nodes connecting to it with a port type other than type- 0 .

Constraints (MT.15) to (MT.23) form the bulk of conditions ensuring that the reachability specified by the $q$-variables are coherent with each other to specify a common tree. These constrains consist in three groups: (MT.15) to (MT.18) for edges between intermediate nodes, (MT.19) to (MT.21) for selecting an edge from the BS, and (MT.22) and (MT.23) for edges between the intermediate nodes and the antennas.

1. Consider first any edge $(i, j)$ between intermediate nodes $i \in \mathcal{N}$ and $j \in \mathcal{N}$. If $(i, j)$ is part of the tree topology (i.e., $\bar{y}_{i j}=1$ ), then the BS and all the antennas are reached in one of the directions $(i, j)$ and $(j, i)$ with one of the port types (possibly zero). This fact is formulated by (MT.15). By (MT.16) and (MT.17), exactly one port type is chosen for each of the two directions of an edge, if the edge is in the tree. For the source BS node $s$, type-0 port must be used in the direction towards it, as stated by constraints (MT.18). Constraints (MT.24) provide consistency in port selection. That is, if arc $(i, j)$ has port type $r$ associated, then this is the only possible port type to be used towards all leaves.

2. Next, consider edge selection towards some intermediate node from the BS. By constraints (MT.19), if edge $(s, j) \in \mathcal{E}$ is selected, then there is one port type associated with $\operatorname{arc}(j, s)$. Furthermore, the port is of type- 0 , as ensured by (MT.20). As a leaf node, the BS is a termination point, thus arc $(j, s)$ does not lead to the other leaf nodes (i.e., the antennas). This fact is explicitly modeled by constraints (MT.21). 
3. The final sets of constraints (MT.22) and (MT.23) model the topology conditions for edges between the intermediate nodes and the antennas. Constraints (MT.22) ensure that if edge $(i, t)$ with $t \in \mathcal{T}$ exists in the solution tree, then a port type other than type0 must be associated with it in the direction from $i$ to $t$. Similar to the source BS, the antennas are leaves and hence termination points of the topology, and, therefore, an arc coming into an antenna does not lead further to any other leaf node, as formulated in constraints (MT.23).

- Power equipment selection: Modeling power equipment selection is relatively simple in DAS-MT. Two sets of constraints, as formulated below, ensure equipment installation and the use of the equipment's port types.

$$
\begin{array}{ll}
\sum_{h \in \mathcal{H}} \bar{z}_{i h}=w_{i}, & \forall i \in \mathcal{N} \\
\sum_{j \in\{s\} \cup \mathcal{N} \cup \mathcal{T}:(i, j) \in \mathcal{A}} q_{i j}^{r j}=\sum_{h \in \mathcal{H}} \alpha_{r h} z_{i h}, & \forall i \in \mathcal{N}, r \in \mathcal{R}
\end{array}
$$

An equipment type is selected for installation at an intermediate node, if and only if the node is in the tree. The condition is formulated with constraints (MT.25). As was mentioned earlier, variable $q_{i j}^{r j},(i, j) \in$ $\mathcal{A}, r \in \mathcal{R}$ has the effect of port selection over arc $(i, j)$ at intermediate node $i$. By taking the sum of the variables over all outgoing arcs for one specific port type, as done in the left-hand side of (MT.26), we obtain the number of ports of type $r$ at node $i$. This has to be equal to the number of ports of the same type that are available on the equipment selected for the node, as all the output ports of the installed equipment should be used. The equality is ensured by (MT.26).

- Path identification: In DAS-F, the path from the BS to every antenna is easily identified by the flow variables. For DAS-MT, the information is carried by the $\bar{x}$-variables. Thus enabling power calculation requires constraints identifying the paths connecting the BS to the antennas, i.e., to link the $\bar{x}$-variables with the topology design. Here, path identification consists in knowing the edges of each path as well 
as the port types along the path. Two scenarios have to be accounted for, as illustrated in the example in Figure 7 for $\operatorname{arcs}(i, j)$ and $(i, k)$ and antenna $t$.

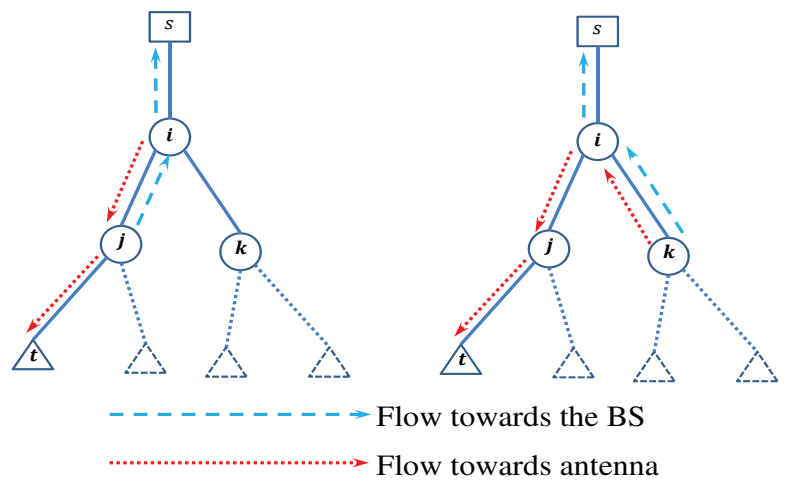

Figure 7: Illustration of path generation.

- Intermediate edge $(i, j)$ is part of the path between the BS and antenna $t$. In this case, one of the two associated arcs $(i, j)$ and $(j, i)$ leads to $t$, and the opposite one leads to the BS. Without loss of generality, suppose $t$ is reached via arc $(i, j)$, see the left part of Figure 7. Thus $\sum_{r \in \mathcal{R}} q_{i j}^{r t}=1$ and $\sum_{r \in \mathcal{R}} q_{i j}^{r s}=0$. For arc $(j, i)$, we have $q_{j i}^{0 s}=1$ since the arc has the direction towards the $\mathrm{BS}$, and $\sum_{r \in \mathcal{R}} q_{j i}^{r t}=0$.

- Intermediate edge $(i, k)$ is not part of the path between the BS and antenna $t$. The scenario is shown in the right part of Figure 7 , assuming without loss of generality that arc $k$ reaches the BS via $i$. In this case, arc $(k, i)$ will also lead to antenna $t$, meaning that $q_{k i}^{0 t}=1$ and $q_{k i}^{0 s}=1$. The opposite arc $(i, k)$ will obviously lead to neither the BS nor antenna $t$, implying $\sum_{r \in \mathcal{R}} q_{i k}^{r t}=0$ and $\sum_{r \in \mathcal{R}} q_{i k}^{r s}=0$.

Based on the above discussion, we introduce the following constraints for linking together the $x$-variables with the tree topology. For edges between the intermediate nodes, the constraints consist of a number of inequalities in order to achieve the desired effect of determining the values of the $\bar{x}$-variables, whereas for the edges of the BS and antennas, the constraints can be formulated as equalities. 


$$
\begin{array}{lll}
\bar{x}_{i j}^{t} \geq \sum_{r \in \mathcal{R}} q_{i j}^{r t}-\sum_{r \in \mathcal{R}} q_{i j}^{r s}, & & \forall(i, j) \in \mathcal{E}: i, j \in \mathcal{N} \\
\bar{x}_{i j}^{t} \leq \sum_{r \in \mathcal{R}} q_{i j}^{r t}+\sum_{r \in \mathcal{R}} q_{i j}^{r s}, & & \forall(i, j) \in \mathcal{E}: i, j \in \mathcal{N} \\
\bar{x}_{i j}^{t} \geq \sum_{r \in \mathcal{R}} q_{j i}^{r t}-\sum_{r \in \mathcal{R}} q_{j i}^{r s}, & & \forall(i, j) \in \mathcal{E}: i, j \in \mathcal{N} \\
\bar{x}_{i j}^{t} \leq \sum_{r \in \mathcal{R}} q_{j i}^{r t}+\sum_{r \in \mathcal{R}} q_{j i}^{r s}, & & \forall(i, j) \in \mathcal{E}: i, j \in \mathcal{N} \\
\bar{x}_{s j}^{t}=\bar{y}_{s j}, & \forall t \in \mathcal{T},(s, j) \in \mathcal{E} \\
\bar{x}_{i t}^{t}=\bar{y}_{i t}, & \forall(i, t) \in \mathcal{E}
\end{array}
$$

Constraints (MT.27) and (MT.29) correspond to the first scenario discussed above (see the left part of Figure 7). If the right-hand side of (MT.27) is one, then arc $(i, j)$ is in the path from $s$ to $t$, hence $\bar{x}_{i j}^{t}$ must be one. Note that the constraints are defined over the edges. Therefore (MT.29) is needed to account for the case that the opposite arc is in the path from $s$ to $t$. Constraints (MT.28) and (MT.30) are formulated for the second scenario (see the right part of Figure 7 ), where edge $(i, j)$ is not in the path between $s$ and $t$. In this case the right-hand side is zero, forcing $\bar{x}_{i j}^{t}$ to be zero as well. Finally, equalities (MT.31) and (MT.32) treats the edges between the BS and intermediate nodes, and those between the intermediate nodes and the antennas.

- Power constraints: Power calculation at the antennas is enabled by combining the $\bar{x}$-variables that identify the paths from the BS and the $q$-variables that carry information of port type of the arcs in the tree. The set of constraints for power calculation is given below.

$$
p_{t}=p_{s}-\sum_{(i, j) \in \mathcal{E}} \xi d_{i j} \bar{x}_{i j}^{t}-\sum_{(i, j) \in \mathcal{A}, r \in \mathcal{R}, i \neq s} r q_{i j}^{r t}, \quad \forall t \in \mathcal{T}
$$

The first sum in (MT.33), $\sum_{(i, j) \in \mathcal{E}} \xi d_{i j} \bar{x}_{i j}^{t}$, represents the total cable loss along the path from the BS to each antenna. The accumulated loss by equipment ports is given by $\sum_{(i, j) \in \mathcal{A}, r \in \mathcal{R}, i \neq s} r q_{i j}^{r t}$. Note that in this sum, 
$q_{i j}^{r t}=1$ does not necessarily mean $(i, j)$ is on the path from $s$ to $t$, as $(i, j)$ can be as well on the path towards $s$, cf. Figure 7 . In this case, however, the port is of type-0 (see the discussion of the constraints for topology design), and hence does not have effect on power calculation. Unlike DAS-F, power calculation by (MT.33) does not require the use of any big-M parameter. Finally, the following inequalities, which are used in DAS-F as well, together formulate maximum allowed power deviation, i.e., $\left|p_{t}^{t a r}-p_{t}\right| \leq p_{t}^{t h r}$.

$$
\begin{array}{ll}
p_{t}^{\text {thr }} \geq p_{t}^{\text {tar }}-p_{t}, & \forall t \in \mathcal{T} \\
p_{t}^{t h r} \geq p_{t}-p_{t}^{t a r}, & \forall t \in \mathcal{T}
\end{array}
$$

\subsection{Objective function}

With the presence of $y$-variables in DAS-MT, expressing the objective of minimizing the total cable length is simple. The function is provided below.

$$
\sum_{(i, j) \in \mathcal{E}} d_{i j} \bar{y}_{i j}
$$

\subsection{Model summary}

Based on the previous discussions, formulation DAS-MT can be summarized as follows:

$$
\begin{array}{rrr}
{[\mathbf{D A S}-\mathbf{M T}]:} & \min & (\mathrm{MT} .36) \\
& \text { s.t. } & (\mathrm{MT} .7)-(\mathrm{MT} .23),(\text { MT.33 }),(\text { MT.34), (MT.35) }
\end{array}
$$

In comparison to DAS-F, DAS-MT is much less straightforward to develop. This increased complexity in modeling does pay off. As will be shown by numerical results, DAS-MT scales much better than DAS-F in problem solving.

\section{Computational efficiency aspects and pre-processing}

IB-DAS deployment optimization is very challenging even for small scenarios. In this section, we discuss problem size reduction within the application context. Namely, real deployment of IB-DAS will follow to a large extent 
engineering practice as well as the restriction posed by the deployment environment. Thus it is reasonable to take these aspects into consideration for the purpose of speeding up the optimization process.

\subsection{Integrating the building structure into modeling}

The computational effort for solving the IB-DAS deployment problem grows rapidly in the size of the underlying graph. Without any restriction on where cable connections can be set up, the edges of the intermediate nodes will form a complete graph. For real-life scenarios, however, this is not the case, because the installation of cables and power equipments, similar to the deployment of other types of electrical infrastructure, will mainly follow the building structure and its internal layout to ease the installation as well as to makes it easier for future maintenance. Furthermore, as an emerging trend, IB-DAS is becoming an integrated part of new buildings' design and construction. Thus the cabling topology will be heavily correlated with building structure. Figure 8 shows a 3D view of one example floor of a building, along with a demonstrative cabling solution of IB-DAS. The cable installation follows well the building structure. In particular, the backbone cables go mainly along the corridors and pathways.

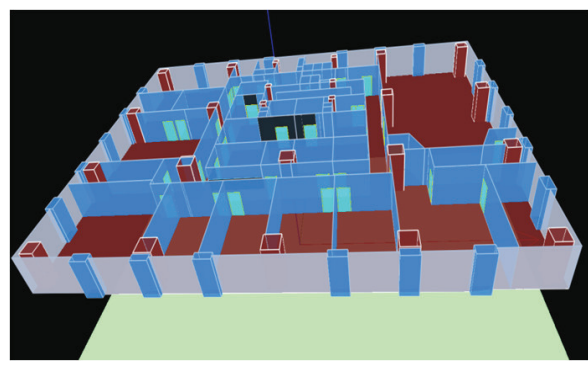

Building structure

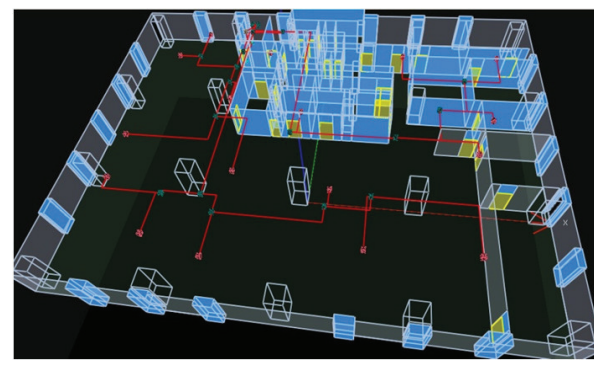

Cable installation

Figure 8: Illustration of cable installation.

As discussed above, we utilize building structure for reducing the problem size of practical IB-DAS deployment. The candidate locations of the intermediate nodes for equipment installation are defined in such a way that they follow the pathways formed by floor layout and that equipment can be easily installed. This pre-processing significantly reduces the number of edges to be considered. An example is shown in Figure 9, with a total of 27 intermediate nodes serving as possible connection points to connect the BS to 11 
antennas. As can be seen, the intermediate nodes give a strong indication of cable installation. In fact, in this example, an underlying tree backbone can be easily identified, though not all intermediate nodes necessarily are part of the optimal deployment and hence the topology optimization task remains.

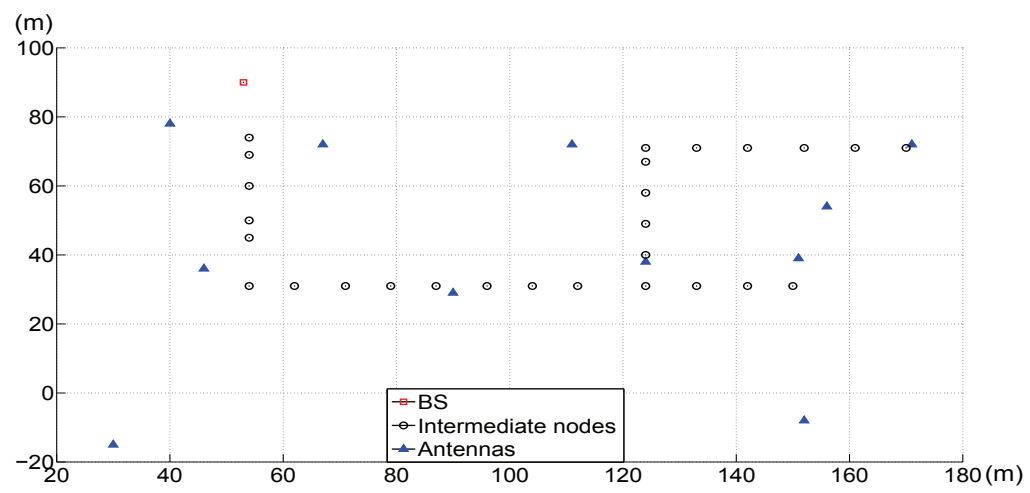

Figure 9: Illustration of the backbone structure of 27 candidate intermediate nodes and 11 antennas.

\subsection{The use of artificial equipment type}

Recall that the artificial equipment type for DAS-MT has two type-0 ports, of which one is simply the opposite direction of the incoming port. The introduction of this equipment type allows for removing edges that can be formed by a sequence of other and shorter edges, as illustrated in Figure 6 . In fact, the idea applies also to DAS-F, by introducing an artificial equipment with one single type- 0 port. The size reduction enables speeding up the optimization process without compromising solution quality.

The size reduction via artificial equipment is significant. In the example of Figure 9, the number of edges between the 27 intermediate nodes is reduced to 26 , i.e., the underlying topology of the intermediate nodes is a spanning tree. A denser scenario with 162 intermediate nodes is shown in Figure 10. In this case, the number of edges of these nodes becomes around 170 after the reduction.

\subsection{Reducing candidate connections for antennas}

Even if connecting an antenna to a far-away intermediate node may be a good choice in theory (e.g., better power output), in practice the antenna 


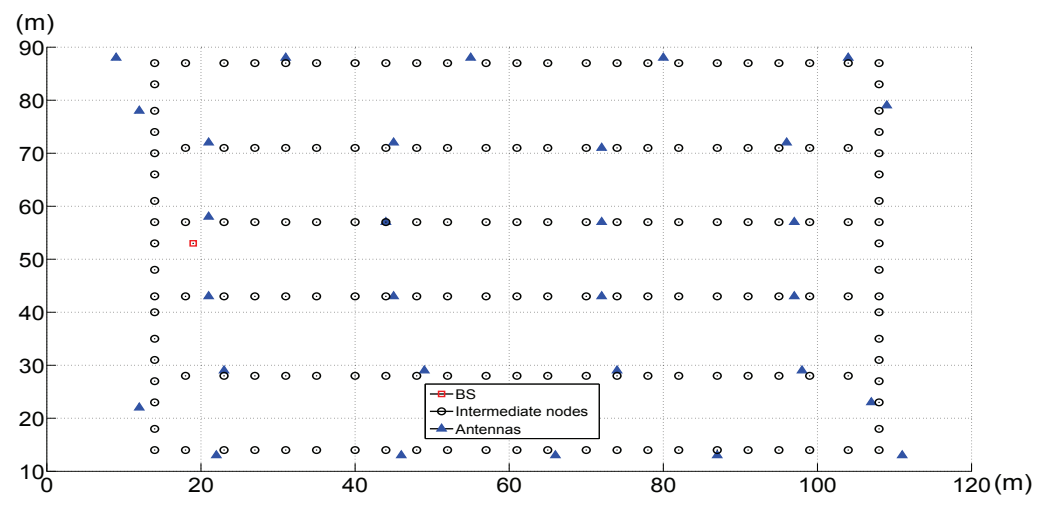

Figure 10: Illustration of the backbone structure of 162 intermediate nodes and 30 antennas.

will be connected to an intermediate node that is nearby. By this engineering practice, pre-processing can be applied to limit the number of candidate edges between the antennas and the intermediate nodes. A similar observation applies to the BS, which is also a leaf node.

In the following, we refer to the candidate intermediate nodes for connecting the antennas and BS into the IB-DAS backbone as leaf-access nodes. For each antenna, it is reasonable to restrict its leaf-access nodes to the $K$ nearest intermediate nodes. For the scenario in Figure 9, the number of leaf-access nodes is reduced from the theoretical $27 \times 11=297$ to 110 if $K=10$, that is, an antenna will be connected to one of the 10 closest intermediate nodes. In the case of Figure 10, the number goes down from 5022 to 310 for the same $K$. Note that if $K$ is too small, the optimization problem becomes infeasible, especially when the power threshold is very stringent. Thus the parameter value is driven by the balance between engineering practice, feasibility, and optimization performance.

\section{Performance evaluation}

\subsection{Planning scenarios}

For performance evaluation, we have used three realistic planning scenarios originating from IB-DAS deployment in two multi-floor buildings. Each scenario involves deploying IB-DAS of one floor. For both buildings, the layouts of the floors and their coverage characterizations are very similar; thus the one-floor solution can be reused at the other floors. Building one has 11 
antennas. For this building, two scenarios are defined, where the numbers of candidate intermediate nodes are 27 and 52, respectively. Building two has 30 antennas. For this building, a scenario with 162 candidate intermediate nodes is defined. The antenna locations as well as the power targets have been derived from a radio access planning tool [21] for IB-DAS. For candidate intermediate nodes, the selection has followed the discussion in Section 6 .

Building layout data have been pre-processed and integrated with the modeling process. The layouts of the first and last scenarios are illustrated in Figure 9 and Figure 10 of Section 6, respectively.

Table 1: A summary of the test scenarios.

\begin{tabular}{rr}
\hline Floor area $\left(m^{2}\right)$ & 10000 \\
Number of candidate intermediate nodes & $27 / 52 / 162$ \\
Number of antennas & $11 / 11 / 30$ \\
Cable loss per meter (dB) & 0.0142 \\
Even power splitters (dB) & $(\mathrm{E} 1: 3.6,3.6),(\mathrm{E} 2: 5.7,5.7,5.7)$ \\
& $(\mathrm{T} 1: 1.5,6.15),(\mathrm{T} 2: 1.95,5.15)$ \\
Uneven power splitters (taps) $(\mathrm{dB})$ & $(\mathrm{T} 3: 0.7,10.15),(\mathrm{T} 4: 1.15,7.15)$ \\
Antenna power target $(\mathrm{dBm})$ & 5 \\
Antenna power deviation threshold $(\mathrm{dB})$ & $1,2,3$ \\
\hline
\end{tabular}

A summary of the scenarios is provided in Table 1. Six equipment types, consisting of two types of power splitters (E1-E2 in the table) and four types of taps (T1-T4 in the table), are available. The power loss settings of these equipment types are given in Table 1 . We apply three antenna power deviation thresholds $(1,2$, and $3 \mathrm{~dB})$, in order to gain insights on the trade-off between computational efficiency and the performance in meeting the power target. The optimization models DAS-F and DAS-MT have been solved using the state-of-art integer programming solver Gurobi [22]. A limit of $10 \mathrm{~h}$ on the computing time is set for each scenario and parameter setting. We first present results for the small scenario of 27 nodes, mainly for the purpose of a comparative study, demonstrating that the non-trivial DAS-MT formulation runs magnitudes faster than the simple formulation DAS-F in reaching the global optimum. Then we focus on the two large scenarios using formulation DAS-MT. After that, we provide a discussion of the time efficiency, and give some general guidelines for practical IB-DAS deployment.

It is always desirable to have result comparison to baseline design solutions in performance evaluation. The IB-DAS problem that we consider, 
however, represents an emerging engineering optimization topic for which there is no well-established algorithmic scheme in the literature. Indeed, the simple IB-DAS design examples in [10] assume that the topology and antenna access are both given and fixed, when equipment selection is performed. This follows the common practice of problem decomposition for wireless network deployment, namely to decompose the problem in question into sequential steps and optimize separately the planning variables of each step.

By the decomposition approach, a baseline scheme for the IB-DAS deployment problem is to first perform topology design, minimizing the total cable length, followed by equipment selection. The first step amounts to solving a degree-constrained minimum spanning tree (D-MST) problem. Note that the presence of the maximum node degree is necessary, as there is a limited maximum number of outgoing ports of the equipment types. As will be illustrated later in Section 7.3, the baseline topological design frequently fails in admitting equipment selection satisfying the power threshold. This result further justifies mathematical modeling integrating the design decisions.

\subsection{Performance results for small-scale scenario}

For the scenario of 27 intermediate nodes in Building one, the number of leaf-access nodes $K$ is set to four and then eight. The two values result in exactly the same optimum, indicating that the number of leaf-access nodes is not critical in this case. Both DAS-F and DAS-MT admit global optimality within the time limit for the cases with thresholds of $3 \mathrm{~dB}$ and $2 \mathrm{~dB}$, while only DAS-MT is able to enable optimum for the case with $1 \mathrm{~dB}$ threshold. The optimal solutions for the three power deviation thresholds $(3,2$, and 1 dB) are shown in Figures 11-13, respectively. The filled black circles indicate the locations of power equipments, and the label (e.g., E1) for each of them represents the equipment type (see also Table 1). One can observe that although the solutions for various power deviation thresholds are similar to each other, differences do occur for antenna connections close to the BS. Note that for two of the three cases, the BS is connected to its second closest intermediate node, as shown by the zoom-in illustration.

The performance results in terms of optimum value (i.e., minimum cable length) and computing times for the case of four leaf-access nodes per antenna are summarized in Table 2. Notation "-" denotes that the time limit of 10 $\mathrm{h}$ is exceeded. The performance figure for the setup of eight access nodes is similar. The computational time values in Table 2 reveal the tremendous difference in the performance of the two formulations, and thereby highlight 


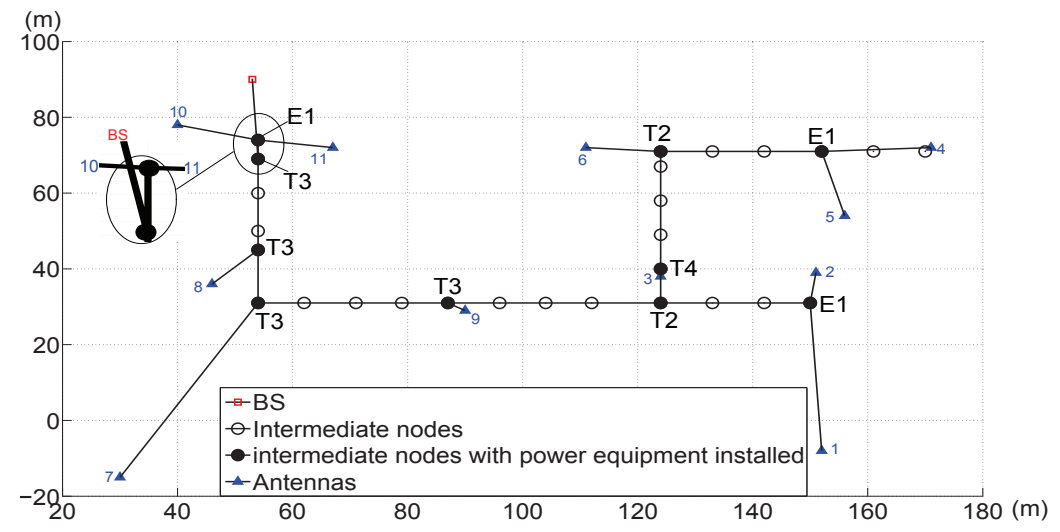

Figure 11: Optimal IB-DAS deployment for the 27-node scenario of Building one with 2 $\mathrm{dB}$ power deviation threshold.

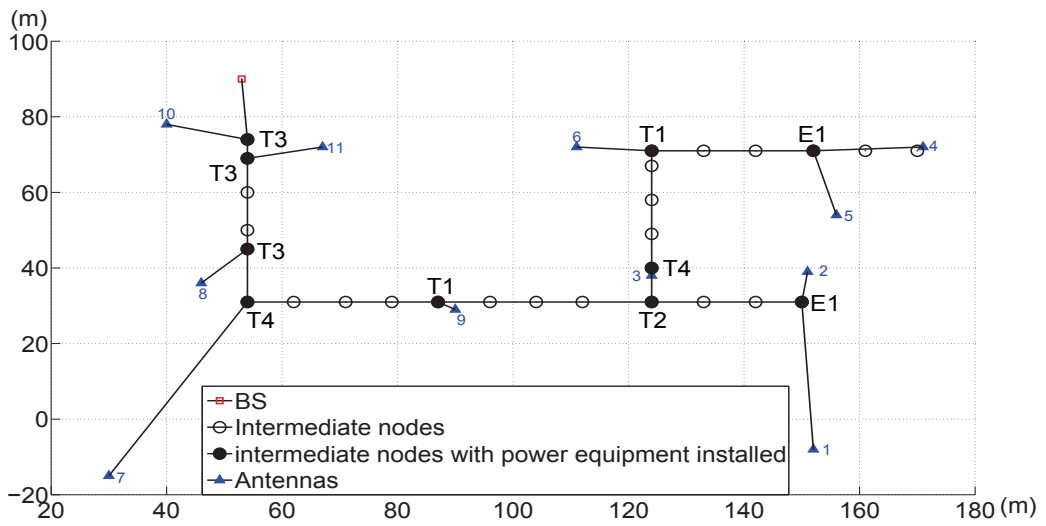

Figure 12: Optimal IB-DAS deployment for the 27-node scenario of Building one with 3 $\mathrm{dB}$ power deviation threshold.

Table 2: Performance results of the 27-node scenario with four leaf-access nodes per antenna.

\begin{tabular}{ccrr}
\hline $\begin{array}{c}\text { Power deviation } \\
\text { threshold }(\mathrm{dB})\end{array}$ & $\begin{array}{c}\text { Optimum cable } \\
\text { length }(\mathrm{m})\end{array}$ & \multicolumn{2}{c}{ Computing time (seconds) } \\
\hline 1 & 465 & - & DAS-F \\
2 & 461 & 27704 & 3 \\
3 & 457 & 123 & 3 \\
\hline
\end{tabular}

the importance of optimization modeling to problem solving. Using DAS$\mathrm{F}$, the computing time jumps from two minutes to several hours, when the 


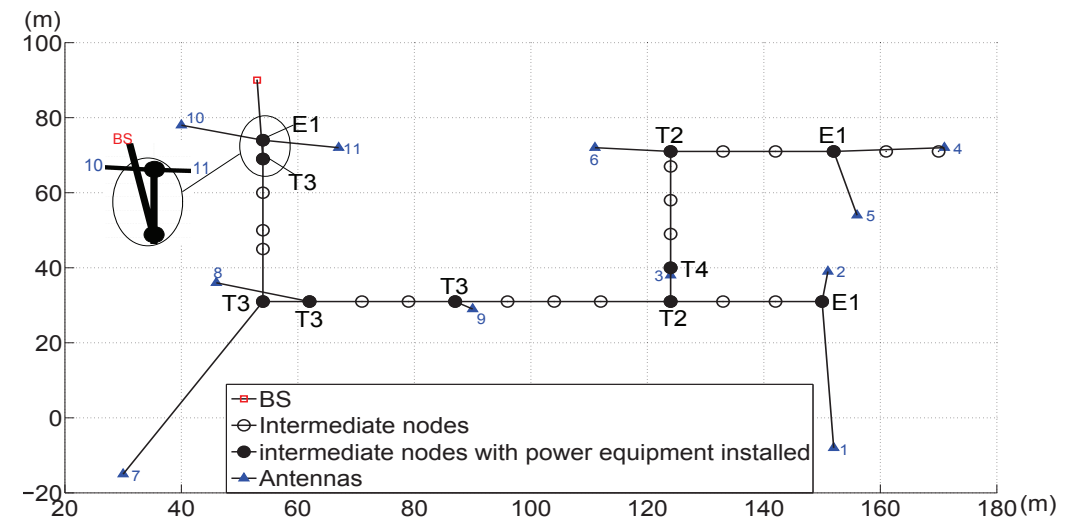

Figure 13: Optimal IB-DAS deployment for the 27-node scenario of Building one with 1 $\mathrm{dB}$ power deviation threshold.

threshold is reduced from $3 \mathrm{~dB}$ to $2 \mathrm{~dB}$. When the most stringent value of $1 \mathrm{~dB}$ is used, optimality is out of reach for DAS-F for the given time limit of 10 hours. In contrast, DAS-MT is able to deliver optimum for all three cases in a matter of few seconds, outperforming DAS-F with several orders of magnitude. Thus our derivation of the less trivial but more structure-tailored formulation DAS-MT does contribute to the solution of IB-DAS deployment. From the table, it is also observed that relaxing the threshold from $1 \mathrm{~dB}$ to $3 \mathrm{~dB}$ allows for less usage of cable, though the difference is minimum.

Being clearly superior to DAS-F in scalability, DAS-MT has a great potential to IB-DAS deployment. In the remainder of the section we focus on the use of DAS-MT to gain insights for larger planning scenarios, along with examining further the computational efficiency aspect.

\subsection{Network deployment solutions for larger planning scenarios}

Formulation DAS-MT is applied to the two larger scenarios, with 52 and 162 candidate intermediate nodes, respectively. For the former, DAS-MT is able to deliver the global optimum for all power deviation thresholds, without any restriction on the number of leaf-access nodes. For the 162-node planning scenario, DAS-MT admits global optimum for some but not all of the cases. Specifically, the formulation yields optimum if the power deviation threshold is 2 or $3 \mathrm{~dB}$ with at most 20 leaf-access nodes per antenna. In Figures 1416 , the deployment solutions for the 52-node scenario with the three power deviation thresholds are illustrated respectively. The solution for the 162node scenario with $2 \mathrm{~dB}$ power threshold and 20 leaf-access node per antenna 
is shown in Figure 17.

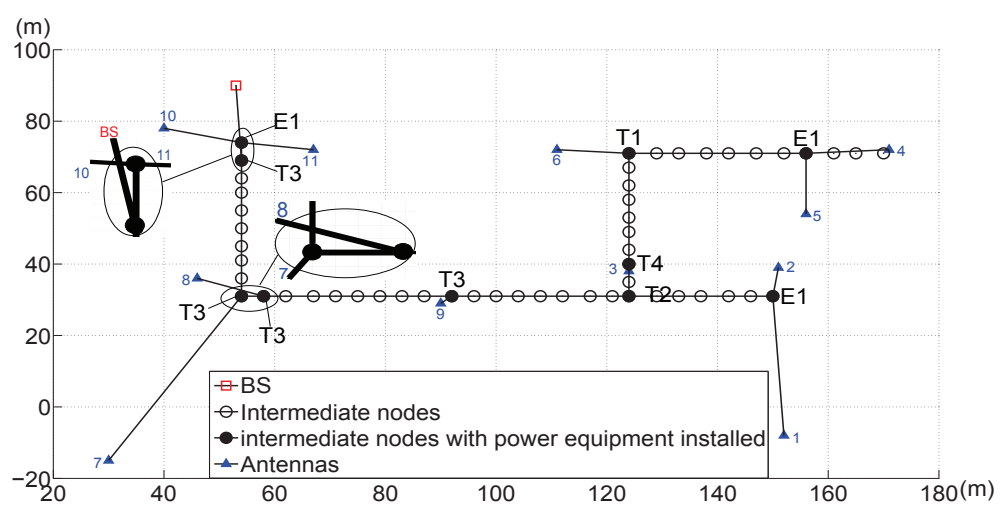

Figure 14: Optimal IB-DAS deployment the 52-node scenario with $1 \mathrm{~dB}$ power deviation threshold.

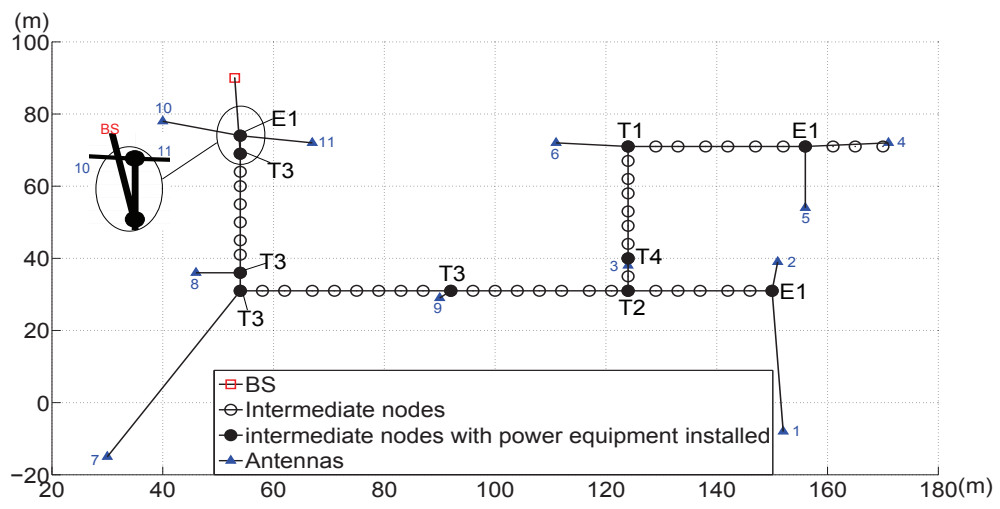

Figure 15: Optimal IB-DAS deployment the 52-node scenario with $2 \mathrm{~dB}$ power deviation threshold.

As can be seen from the 52-node scenario, varying the power deviation threshold leads to slightly different topology solutions and power equipment type selections. There is clear coupling between the threshold and topology, namely the selection of leaf-access nodes becomes less intuitive for smaller threshold in order to satisfy the threshold constraint. An example is antenna eight. This antenna is connected to its closest intermediate node when the threshold is over $2 \mathrm{~dB}$, whereas for the $1 \mathrm{~dB}$ threshold an intermediate node being further away is chosen, For the 162-node scenario, the underlying 


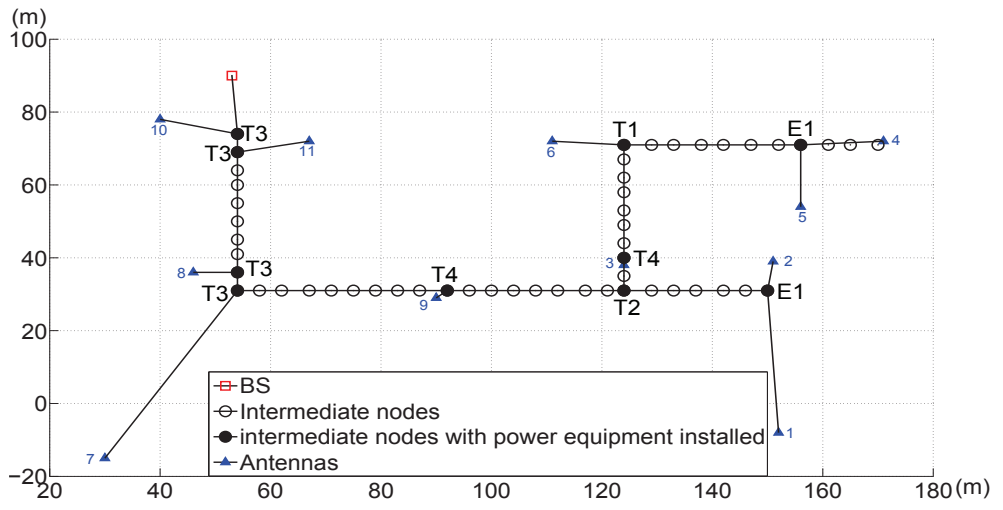

Figure 16: Optimal IB-DAS deployment the 52-node scenario with $3 \mathrm{~dB}$ power deviation threshold.

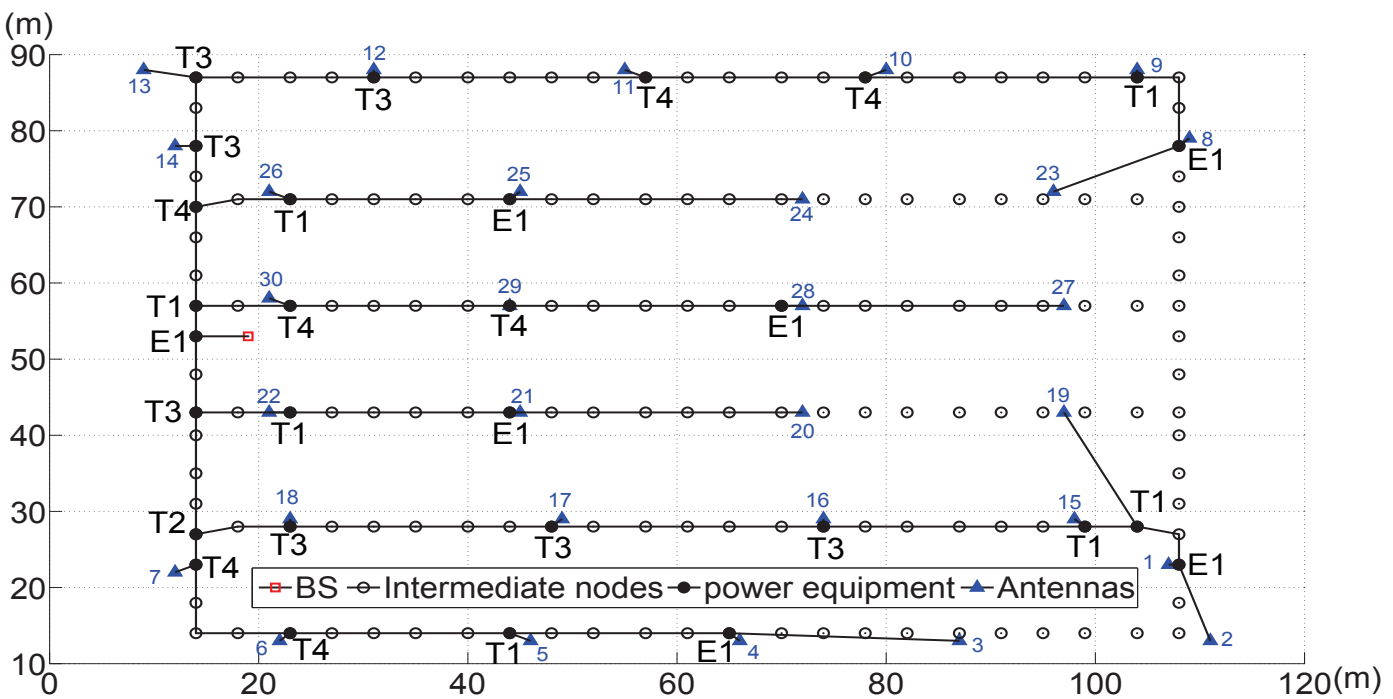

Figure 17: Optimal IB-DAS deployment for the 162-node scenario with 2 dB power deviation threshold.

topology is denser. As a result, a rather significant number of the intermediate nodes are not used, as can be observed in Figure 17. The equipment type of even-power splitter with three ports is not present in any of the optimal design solutions. The explanation originates from the regular building structure, for which it is hardly feasible to have three branches at any intermediate node.

In Figures 14-17, the main layout of cabling is quite intuitive. Thus 
the solutions may appear to submit to a manual approach of simply setting up the design by hand. However, two aspects prohibit the use of such an approach. First, for a large-scale scenario, determining the optimal links connecting the intermediate nodes is not simple. For example, in Figure 17 two cables terminate at antennas 20 and 24, respectively, even if there do exists additional "downstream" antennas to be connected. In contrast, the cable in the middle goes all the way to antenna 27 . This optimal combination of connections does not follow directly from manual planning. Second and more importantly, the approach is very prone to errors in selecting antenna access, i.e., to which intermediate node each antenna should be connected, because in many cases the optimal choice is not the intuitive one. Examples include the zoomed-in areas in Figures 14-15, and antennas 19 and 23 in Figure 17. Note also that the power output at an antenna depends on the equipment selection along the entire path to the BS. Thus the performance is a joint effect of topology design and equipment selection, and decoupling these decisions does not result in a feasible solution scheme.

In Figures 18-19, we provide the distribution of the antenna power for the design solutions. The labels on the $\mathrm{x}$-axis represent antenna indices. The $\mathrm{y}$ axis is the antenna power, with a target value of $5 \mathrm{dBm}$ for all antennas. From the figures, one observes that the proportion of antennas having the maximum allowed deviation is about $10 \%$, hence most of the power deviations are strictly within the threshold. As expected, the power deviation tends to grow when the threshold increases. For the 52-node scenario, the average power deviations over the antennas are $0.58,0.83$, and $1.74 \mathrm{~dB}$ for thresholds of 1,2 , and $3 \mathrm{~dB}$, respectively. For the 162-node scenario, the average value is 1.05 for the $2 \mathrm{~dB}$ threshold. Thus the overall deviation is about half of the specified threshold value.

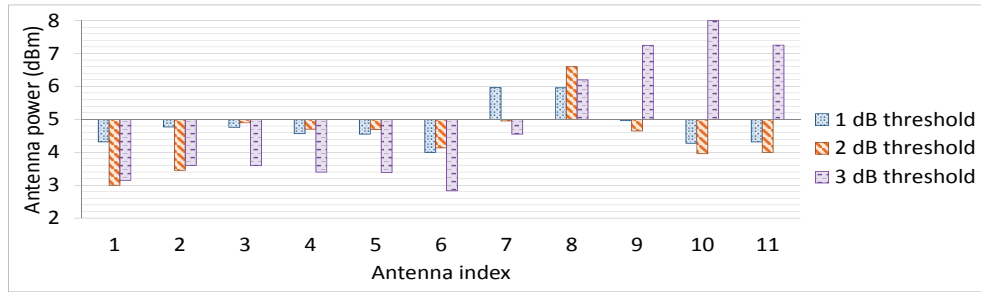

Figure 18: Antenna power distribution for the 52-node scenario. 


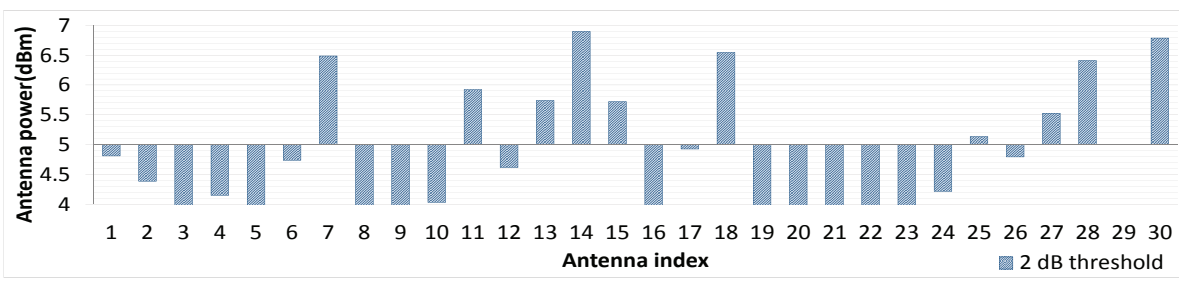

Figure 19: Antenna power distribution for the 162-node scenario with threshold $2 \mathrm{~dB}$.

\subsection{Computational performance aspects}

From a performance viewpoint, several aspects are of significance in computational optimization of IB-DAS deployment. First, to approach global optimality, the choice of optimization formulation is crucial, as demonstrated by the comparative study in Section 7.2. Second, the problem size, in particular the number of edges has high impact on the computational requirement. Utilizing the backbone structure derived via building survey, as was illustrated in Section 6, is a key step towards computational efficiency. The number of edges is also highly dependent on the number of leaf-access nodes (i.e., parameter $K$ ) defined for each antenna. Reducing the number speeds up the optimization process but potentially has a negative impact on the total cable length or even causes infeasibility. Third, decreasing the power deviation threshold will drive the solution closer to the power target. At the same time, it becomes harder to solve the optimization problem, which may be infeasible if the threshold is too small.

In Table 3, we summarize the performance results of solving the IBDAS deployment problem for the three scenarios by DAS-MT for various combinations of $K$ and power deviation thresholds. In the table, "-" indicates that no solution is obtained within the time limit.

As can be observed from the table, the graph size in the number of edges grows with respect to the number of leaf-access nodes, and the amount of growth increases by scenario size. For the 27 -node scenario, setting $K=20$ instead of 10 gives a moderate size increase. If the restriction is waived completely (i.e., an antenna can be connected to any of the intermediate nodes), the number of edges is more than doubled. For the largest scenario of 162 nodes, increasing $K$ from 10 and 20 brings the number of edges from 471 to 781. If no restriction is imposed, the number jumps to above 5000 . The increase in graph size directly translates to the computational effort, particularly if the power deviation threshold is small. Consider the threshold 
Table 3: A summary of performance results.

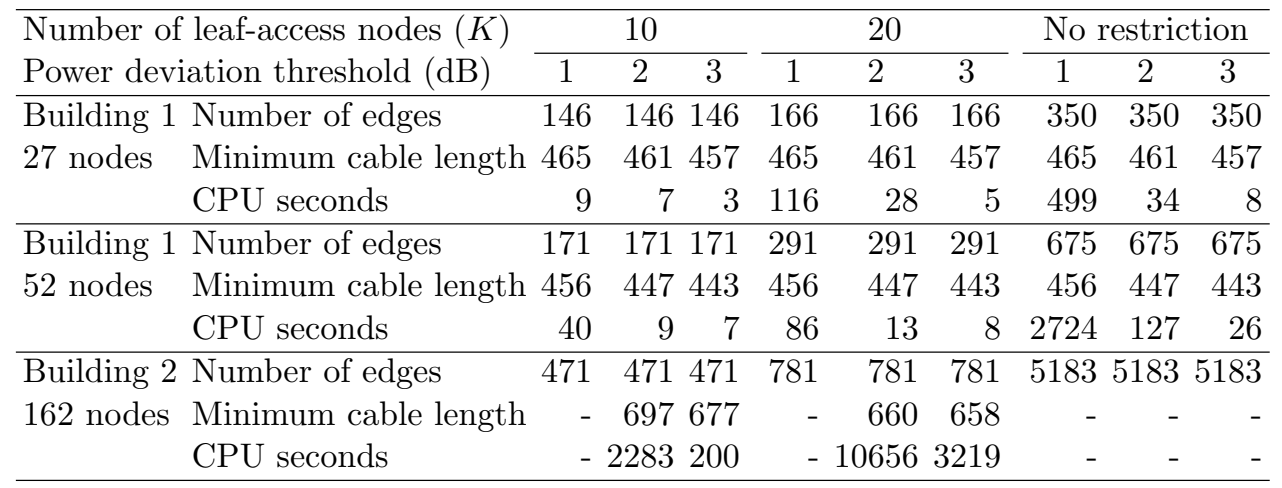

of $2 \mathrm{~dB}$. Allowing complete freedom of leaf access forces the computing time to grow by the order of about one magnitude in comparison to the case of 10 leaf-access nodes per antenna.

The power deviation threshold also has significant influence on the computing time. Consider the first two scenarios with the setting of no restriction on leaf-access nodes. Setting a threshold of $3 \mathrm{~dB}$ requires 8 and 26 seconds for the two scenarios, respectively. Reducing the threshold to $2 \mathrm{~dB}$, the solution times become much longer, while setting the threshold further down to $1 \mathrm{~dB}$ requires another order of magnitude of increase in computing effort. The impact of power deviation threshold is intertwined with that of graph size. As can be seen for the 162-node scenario, the trend in respect of power deviation is amplified in the results. The magnitude of computing time moves up by an order when the threshold is decreased from 3 to $2 \mathrm{~dB}$, and a threshold of $1 \mathrm{~dB}$ does not admit any solution either because of the time limit or because no feasible solution exists.

Next, we examine the effect of $K$ and power deviation threshold on the performance metric of total cable length. For the first two scenarios, limiting the number of leaf-access nodes has no impact at all, meaning that the computational efficiency can be significantly improved without compromising solution quality. For the last scenario, doubling the number of leaf-access nodes improves the total cable length by $3-4 \%$. Thus the impact of $K$ appears small, indicating that limiting the choice of leaf access is reasonable both in view of the engineering practice and the benefit of problem size reduction. For all three scenarios, the total cable length improves consistently 
by relaxing the power deviation constraint. However, the difference is very moderate. Thus setting the power deviation threshold shall be based on the importance of meeting the power target and computational aspects, rather than its impact on the optimization objective.

In Table 3, there are a number of cases for which no solution is obtained for the 162-node scenario. For the threshold values of 2 and $3 \mathrm{~dB}$, the lack of solution is clearly caused by problem size, as the problem could be successfully solved by restricting the number of leaf-access nodes. Note that any solution found with restricted leaf access $(K=10$ and $K=20)$ is also a valid design for the same scenario with no restriction on $K$. Therefore feasible though not necessarily optimal solutions are indeed available for the two thresholds under column 'No restriction' in Table 3. For the most stringent threshold of $1 \mathrm{~dB}$, the reason of no solution in the table could be problem infeasibility, namely the solution space is in fact empty.

From the results and discussions, setting the number of leaf-access nodes as well as the power deviation threshold are of high importance in IB-DAS planning. For a specific planning case, a number of computations may be performed in order to balance the computation and performance.

Table 4: Performance results of the baseline design.

\begin{tabular}{lcc}
\hline Scenarios & $\begin{array}{c}\text { Cable } \\
\text { length }(\mathrm{m})\end{array}$ & $\begin{array}{c}\text { Minimum achievable } \\
\text { power deviation }(\mathrm{dB})\end{array}$ \\
\hline Building 1, 27 nodes & 457 & 2.31 \\
Building 1, 52 nodes & 443 & 2.31 \\
Building 2, 162 nodes & 657 & 3.35 \\
\hline
\end{tabular}

In Table 4, we report the performance results of the baseline design scheme described in Section 7.1, namely, to decompose the problem into two sequential stages of topology optimization and equipment selection, respectively. The topology is derived from solving degree-constrained MST. For the given D-MST solution, we solve the equipment selection problem, with the cost function being the power deviation from the target. Thus the optimum value is the minimum achievable power deviation. If the value is within the interval for the threshold, equipment selection is feasible and the D-MST topology is in fact globally optimal, otherwise the scheme fails. For the first two scenarios in Building one, the baseline topology admits equipment selection for the threshold of $3 \mathrm{~dB}$, and hence the topology solutions coincides 
with the corresponding ones in Table 3. When the threshold decreases, however, no feasible equipment selection exists for the baseline topology. For the last scenario, the baseline topology fails, even for the largest power deviation threshold. The results reveal that topology design is strongly intertwined with equipment selection in determining the overall performance, demonstrating the advantage of mathematical model that takes a global view of the problem.

\section{Conclusions}

By bringing radio access closer to the end users, IB-DAS is a promising solution for coverage and capacity for in-building mobile broadband. In this paper, we have studied optimization of passive IB-DAS deployment. The resulting optimization problem is very challenging. Topology design and equipment installation, driven by the cable cost and the power targets of the antennas, are tightly intertwined in the optimization process. We have taken an integer programming approach with emphasis on mathematical modeling. Two types of models, DAS-F and DAS-MT, have been developed. DAS-F, based on the widely used concept of network flows, is straightforward but proven to be useful only for small planning scenarios. DAS-MT uses the concept of embedding multiple trees into a common tree. The formulation is more complicated than DAS-F, but exhibits high scalability. In addition, pre-processing techniques, ranging from integrating building structure to the notion of artificial equipment, have been developed for improving computational efficiency.

The numerical results demonstrate that the solution approach of modeling and mathematical programming is capable of approaching global optimality with reasonable computing effort for planning scenarios of practical relevance. Our computational study also sheds light on the trade-offs between optimality, computational efficiency, and network performance. As a future work, fast heuristics that are able to finding feasible solutions to very large scale IB-DAS deployment call for investigation, within which the results of the current paper effectively serve the purpose of benchmarking in performance evaluation. Algorithms that target determining problem feasibility for given power deviation threshold form another line of forthcoming research. Moreover, we remark that the DAS deployment problem considered in the current paper is defined for given antenna locations and candidate interme-

diate nodes, though for the latter the optimization process has to determine 
which will actually be used in a design solution. Even with the locations given, approaching global optimum is challenging for large scale cases, as can be observed from the computational results. Extending the problem setting, in particular to include antenna location in the optimization process, is of practical interest, and solution approaches for the joint optimization problem form an important line of future work.

\section{Acknowledgment}

We would like to thank the anonymous reviewers for their valuable comments and suggestions. The work has been partially supported by the ELLIIT research excellence center of Sweden, CENIIT of Linköping University, Sweden, and the EC FP7 Marie Curie Programme.

\section{References}

[1] Nokia Siemens Networks, "In Building Solutions Executive Summary," 2011.

[2] D. Hong, S. Choi, and J. Cho, "Coverage and capacity analysis for the multi-layer CDMA macro/indoor-picocells," in IEEE International Conference on Communications (ICC'99), vol. 1, pp. 354-358, 1999.

[3] J. Perez-Rornero, O. Salient, and R. Agusti, "On the capacity degradation in WCDMA uplink/downlink due to indoor traffic," in IEEE 60th Vehicular Technology Conference (VTC2004-Fall), vol. 2, pp. 856-859, 2004.

[4] K. Hiltunen, B. Olin, and M. Lundevall, "Using dedicated in-building systems to improve HSDPA indoor coverage and capacity," in IEEE 61st Vehicular Technology Conference (VTC2005-Spring), vol. 4, pp. 23792383, 2005.

[5] B. Song, R. Cruz, and B. Rao, "Downlink optimization of indoor wireless networks using multiple antenna systems," in 23rd Annual Joint Conference of the IEEE Computer and Communications Societies (INFOCOM'04), vol. 4, pp. 2778-2789, 2004.

[6] H. Beijner, "The importance of in-building solutions in third generation networks," Ericsson Review, 2004. 
[7] J. Borkowski, J. Niemela, T. Isotalo, P. Lahdekorpi, and J. Lempiainen, "Utilization of an indoor DAS for repeater deployment in WCDMA," in IEEE 63rd Vehicular Technology Conference (VTC2006-Spring), vol. 3, pp. 1112-1116, 2006.

[8] L. Chen, D. Yuan, H. Song, and J. Zhang, "Mathematical Modeling for Optimal Deployment of In-Building Distributed Antenna Systems," in the first International Conference on Communications in China (IEEEICCC), pp. 786-791 2012.

[9] "The DAS forum." www.thedasforum.org

[10] M. Tolstrup, Indoor Radio Planning, A Practical Guide for GSM, DCS, UMTS and HSPA. John Wiley \& Sons, Ltd, 2008.

[11] M. R. Garey and D. S. Johnson, Computers and Intractability: A Guide to the Theory of NP-Completeness, W. H. Freeman, 1979.

[12] R. Rardin and L. Wolsey, "Valid inequalities and projecting the multicommodity extended formulation for uncapacitated fixed charge network flow problems," Université catholique de Louvain, Center for Operations Research and Econometrics (CORE), 1990.

[13] D. Bienstock and O. Günlük, "Capacitated network design - polyhedral structure and computation," Informs Journal on Computing, vol. 8, pp. 243-259, 1994.

[14] J. Hellstrand, T. Larsson, and A. Migdalas, "A characterization of the uncapacitated network design polytope," Operations Research Letters, vol. 12, pp. 159-163, 1992.

[15] P. Belotti, F. Malucelli, and L. Brunetta, "Multicommodity network design with discrete node costs," Networks, vol. 49, pp. 90-99, 2007.

[16] D. Yuan, J. Bauer, and D. Haugland, "Minimum-energy broadcast and multicast in wireless networks: An integer programming approach and improved heuristic algorithms," Ad Hoc Networks, vol. 6, pp. 696-717, 2008.

[17] J. Bauer, D. Haugland, and D. Yuan, "Analysis and computational study of several integer programming formulations for minimum-energy 
multicasting in wireless ad hoc networks," Networks, vol. 52, pp. 57-68, 2008.

[18] M. Pioro and D. Medhi, Routing, Flow, and Capacity Design in Communication and Computer Networks, Morgan Kaufmann Publishers, 2004.

[19] B. N. Khoury, P. M. Pardalos, and D. Hearn, "Equivalent formulations for the steiner problem in graphs," D. Z. Du et al, (eds.), Network Optimization Problems, pp. 111-124, 1993.

[20] T. Polzin and S. V. Daneshmand, "A comparison of steiner tree relaxations," Discrete Applied Mathematics, vol. 112, pp. 241-261, 2001.

[21] RANPLAN iBuildNet@, RANPLAN. www.ranplan.co.uk

[22] Gurobi solver, Gurobi Optimization. www.gurobi.com 